\title{
Ultrafast adiabatic frequency conversion using slow-light in photonic crystal waveguides
}

\author{
D. M. Beggs*a, T. Kampfrath ${ }^{\mathrm{b}}$, L. Kuipers ${ }^{\mathrm{b}}$, T. F. Krauss ${ }^{\mathrm{a}}$ \\ ${ }^{a}$ School of Physics \& Astronomy, University of St Andrews, KY16 9SS UK; \\ ${ }^{\mathrm{b}}$ FOM Institute AMOLF, Science Park 104, 1098 XG Amsterdam, The Netherlands
}

\begin{abstract}
By performing an ultrafast pump-probe experiment, we demonstrate the adiabatic frequency conversion of a telecommunications pulse in a silicon photonic crystal waveguide. By using slow-light modes to spatially compress the pulse, a 1.3 ps long pulse is blue-shifted by $0.3 \mathrm{THz}$ with $80 \%$ efficiency in a waveguide just $19 \mu \mathrm{m}$ long. We also present the results of an adiabatic model of the process, which agrees excellently with the experimentally measured data.
\end{abstract}

Keywords: ultra fast, pump-probe, slow light, silicon, photonic crystals, W1, waveguide

\section{INTRODUCTION}

Future terabit per second telecommunications networks will increasingly operate all-optically, removing the bottleneck of electro-optic conversion that is currently required. Also, as computer chips become massively parallel with multiple cores, on-chip optical communications becomes attractive as compared to the electronic equivalent. Both of these key technologies require that optical network components such as routers, modulators and frequency converters be integrated on-chip. A further requirement is that they are made from silicon [1-9], ensuring CMOS compatibility.

However, silicon photonics suffers from the very weak non-linear effects and small refractive index changes available, especially when compared to III-V semiconductor materials. One solution is to enhance the sensitivity of light to the small refractive index changes using a resonant effect.

Slow-light is a resonant effect that occurs in periodic structures, such as coupled resonator optical waveguides and photonic crystals. In these periodic structures, the forwards and backwards travelling components of the wave interfere to give a standing wave (with group velocity $v_{g}=0$ ) at the bandedge created by the periodicity. Just away from the bandedge, however, there is a region of slow light $\left(v_{g}<<c\right)$, where the envelope of the interfering backwards and forwards travelling components moves forwards only very slowly.

Recently we have demonstrated the application of slow-light enhancement to silicon photonic crystal switches and modulators $[10,11]$, including the ultrafast demonstration of a 3 ps switching time [12]. Here we further demonstrate the adiabatic frequency conversion of a telecommunications pulse using a slow-light enhanced photonic crystal waveguide fabricated in a silicon slab and just $19 \mu \mathrm{m}$ in length. A frequency shift of $0.3 \mathrm{THz}$ with an $80 \%$ power conversion efficiency is demonstrated in an ultrafast pump-probe experiment for a 1.3 ps input pulse at telecommunications wavelengths [13]. Such a frequency shift is sufficient for moving a signal over $\sim 10$ dense WDM channels.

\section{EXPERIMENTAL DESIGN}

The frequency conversion is demonstrated using the slow-light modes of a W1 photonic crystal waveguide. A W1 waveguide is a single row of missing holes from an hexagonal lattice of holes perforating a silicon slab as shown schematically in fig. 1 . The W1 waveguide system has undergone extensive study [14-17], and the functional form of its dispersion ( $\omega$ vs. $k$ ) relation is well known.

Our W1 waveguide is just $19 \mu \mathrm{m}$ long and has holes of diameter $277 \mathrm{~nm}$ and a lattice constant of $390 \mathrm{~nm}$ in a silicon slab of thickness $220 \mathrm{~nm}$. We have calculated the dispersion relation in fig. 2, and it can be seen from the flat slope of the dispersion curve that there is a slow-light mode at a frequency of around $202 \mathrm{THz}$. We launch a pulse into this slowlight mode and, whilst the pulse is inside the waveguide, perform a frequency shift on the eigenfrequency of the mode by altering the refractive index of the whole silicon slab by a small amount $\Delta n$. This perturbation causes the 
eigenfrequencies of the system to blue-shift, as illustrated in fig. 1 (a). If a light pulse is present in the waveguide at the time of the perturbation, it will also undergo a blue-shift along with the modes.

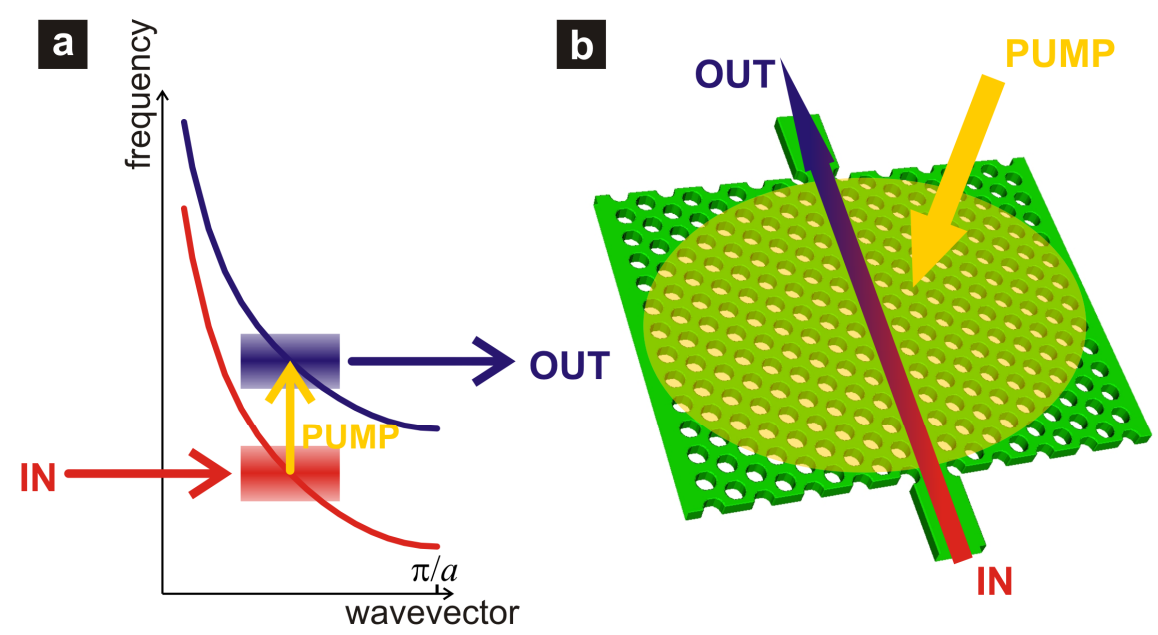

Figure 1. Schematic illustration of the experiment, showing the dispersion relation ( $\omega$ vs. $k$ ) of the modes (a) and the structure of the waveguide (b). A pulse is coupled into the W1 photonic crystal waveguide mode (red line), and, whilst the pulse is inside, the waveguide is hit with a pump-pulse leading to the perturbation of the system (as described in the text). The pump-induced blue-shifted waveguide mode is shown in blue, and the original input pulse is blue-shifted before being output.

In order to provide the $\Delta n$, we illuminate the whole W1 waveguide from above with an ultrafast laser pump-pulse of duration $150 \mathrm{ps}$ and centre wavelength $800 \mathrm{~nm}$, as illustrated in fig. 1 (b). The absorption of this pump pulse by the silicon layer leads to an abrupt change downwards of the refractive index due to the creation of free electron-hole pairs. For example, absorbing approximately $6 \mathrm{pJ}$ of the pump pulse leads to a $\Delta n=-7.7 \times 10^{-3}$, which we have calculated causes the blue-shift of the modes by $0.39 \mathrm{THz}$, a value which is independent of the wavevector $k$. The W1 waveguide is clad in silica, in order to assist with the dissipation of the heat also caused by the absorption of the pump pulse.

If the perturbation acts more slowly than the inverse frequency spacing between the mode and the next available mode, then the system will evolve adiabatically, and the light inside the waveguide at the time of the perturbation will have been efficiently shifted to the new eigenfrequency. In other words, no power transfer between modes can occur unless the perturbation is faster than the inverse frequency spacing. Since we excite the entire silicon slab, the pump pulse respects the symmetry of the photonic crystal waveguide, and there is no transfer of momentum between the pump and the probe. That is to say: only vertical transitions on the dispersion diagram in fig. 2 are allowed in non-adiabatic processes. As can be seen from fig. 2, the next available mode is over $5 \mathrm{THz}$ away, meaning that perturbations as fast as $\sim 150$ fs can give rise to adiabatic processes.

Although we have used the slow-light modes of a photonic crystal waveguide, we would like to emphasize that neither the slow-light nor the photonic crystal are fundamentally required for the frequency conversion of the pulse. Indeed, the experiment, in principle, could be performed in a simple silicon slab or even in bulk material.. In practice, however, the demonstration of adiabatic and efficient frequency conversion in bulk material would be very difficult for two reasons.

Firstly, any pulse traveling in fast modes will be spatially large, meaning that the perturbation would need to act over a large volume of silicon, as only that fraction of the pulse with overlap with the perturbation will undergo frequency conversion. Thus the efficiency of the shift will be reduced and/or the energy required increased. The slow-light modes spatially compress the pulse, so that it can be held in a small volume of silicon whilst the perturbation acts on it in its entirety. Secondly, the frequency spacing between modes would be small, requiring the perturbation to be slow, and any pulse would be able to travel large distances during this time. Therefore the photonic crystal or waveguide structure is used for its large mode spacing, enabling both an ultrafast perturbation and adiabatic dynamics. Also the photonic crystal waveguide has advantages as compared to both a cavity (which cannot host a complete pulse) [18, 19] and a normal waveguide (whose modes are too fast for a small device). 


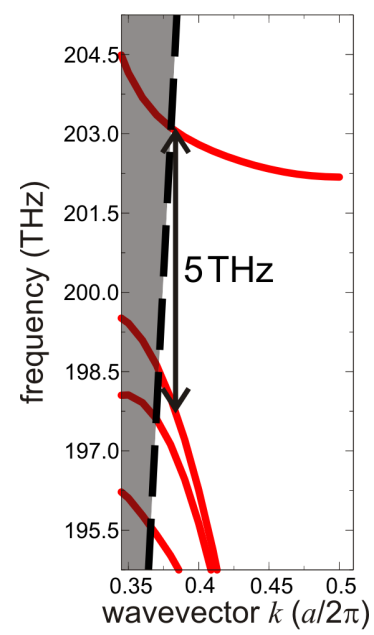

Figure 2. Dispersion relation of the W1 photonic crystal waveguide, calculated for the parameters as described in the text. The slow-light waveguiding mode can be seen around $202 \mathrm{THz}$. The light-line of the silica cladding is indicated by the dashed black line.

\section{FABRICATION OF THE PHOTONIC CRYSTAL WAVEGUIDES}

The fabrication of the photonic crystal waveguides closely follows that given in refs. [20] and [21, 22]. A silicon-oninsulator wafer (SOITEC) with a $220 \mathrm{~nm}$ thin silicon membrane on a $2 \mu \mathrm{m}$ thick buried oxide later is used as the starting point. The $\mathrm{W} 1$ waveguide is formed by patterning the silicon layer with holes by electron-beam lithography - a $400 \mathrm{~nm}$ layer of ZEP-520A (Zeon Chemicals) is used as a resist and etch mask, and the pattern is transferred from the mask to the silicon layer using reactive ion etching with a $\mathrm{SF}_{6} / \mathrm{CHF}_{3}$ gas mixture.

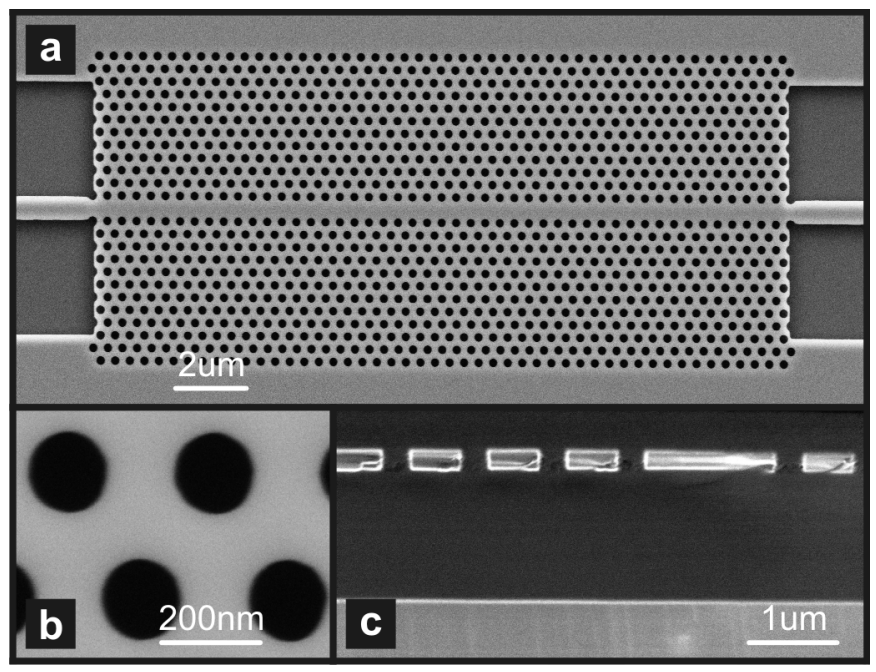

Figure 3. Fabrication of the W1 photonic crystal waveguides. (a) SEM images of the W1 waveguide after the transfer of the hole pattern to the silicon layer, but before the silica top-cladding is deposited. The silicon slab waveguides used as input to and output from the W1 can also be seen. (b) Close-up of the holes perforating the silicon membrane. (c) Cross-section of the final device, after the deposition of the silica top-cladding. The layer structure is (from the top) - silica top-cladding $(550 \mathrm{~nm})$, silicon layer $(220 \mathrm{~nm})$ perforated with holes, silica bottom cladding $(2 \mu \mathrm{m})$, and finally the silicon substrate.

Figure 3 (a) shows a scanning electron microscope (SEM) image of the fabricated W1 waveguide in the silicon layer. Slab waveguides, used for input and output from the W1 waveguide are also visible. Figure 3 (b) shows a close-up SEM image of the holes in the silicon slab that make up the W1. After the transfer of the hole pattern to the silicon layer, the resist and mask is removed and a silica cladding is then deposited using the spin-on-glass Fox-14 (Dow-Corning), which 
leaves a $550 \mathrm{~nm}$ thick layer after hard baking. A cross-section of the final device is shown in fig. 3 (c), where the silica cladding can be seen to infill the holes in the silicon slab.

\section{ULTRAFAST PUMP-PROBE CHARACTRISATION}

After fabrication of the W1 waveguides, we then measured the output of the probe pulse as a function of the delay $\tau$ between the probe and the pump. By interfering the output with a well characterized reference pulse, we can measure both spectral phase and intensity of the output waveform.

Figure 4 (a) and (b) respectively show the output intensity and group delay (the derivative of the phase) when a broadband probe-pulse (duration $180 \mathrm{fs}$ FWHM) is used as input for two example values of the delay, namely $\tau=-5 \mathrm{ps}$ (red curves), meaning the probe arrives long before the pump and experiences the waveguide in its ground state, and $\tau=$ +5 ps (blue curves), when the probe arrives long after the pump and sees an excited waveguide. The spectrum of the broadband input is also shown in fig 4 (b).

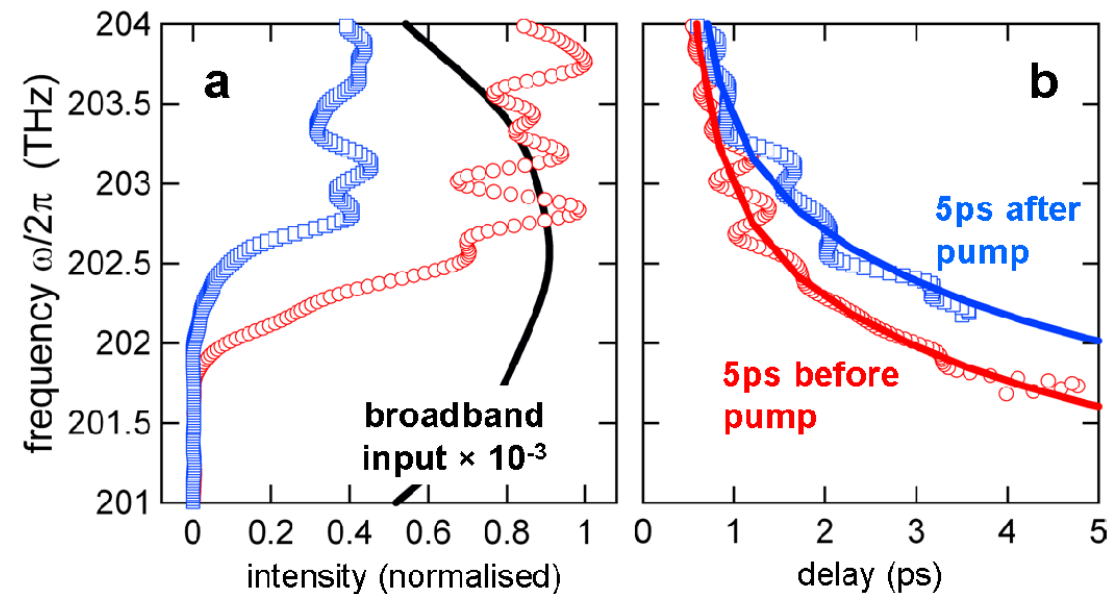

Figure 4. Characterisation of the time-varying W1 photonic crystal waveguide. Measured output spectra (a) and group delay (b) when the broadband pulse shown by the black line in (a) is used as input. Two example delays are shown: the probe arrives 5 ps before the pump ( $\tau=-5 \mathrm{ps}$, red dots), and 5 ps after the pump ( $\tau=+5$ ps, blue dots). The solid lines in (b) also show the calculated phase delays for an unperturbed W1 waveguide (red) and one perturbed with a $\Delta n \stackrel{\sim}{=} 7.7 \times 10^{-3}$. The pump-induced blue-shift of the eigenmodes is clearly seen.

The transmitted intensity after arrival of the pump pulse [blue line in fig. 4 (a)] is around half that of the value before (red line). This is due to the absorption of the probe light by the free carriers generated by the arrival of the pump. Also the cut-off frequency of the W1 waveguide [17] is seen to be blue-shifted by the arrival of the pump. This blue-shift is confirmed in fig. 4 (b), which also the phase delays calculated for the original structure and one perturbed by a $\Delta n=-7.7$ $\times 10^{-3}$ (solid lines), as described in section 2. An excellent fit is obtained for both $\tau= \pm 5 \mathrm{ps}$, so it can clearly be seen that the arrival of the pump pulse blue-shifts the eigenfrequencies of the W1 waveguide by $0.39 \mathrm{THz}$.

Once we have measured the complex valued output of the broadband probe pulse as a function of the pump-probe delay $\tau$, we have sufficient information to extract the output of any input pulse without the need of further experiments. The only condition is that the spectrum of this input pulse is contained in the spectrum of the broadband input [fig. 4 (a)]. Details on this procedure can be found in ref. [23]. In this way, we have investigated the output of a 1.3 ps long bandwidth-limited probe-pulse centred at $202 \mathrm{THz}$. The results are shown in fig. 5 (a).

For $\tau<-3 \mathrm{ps}$, the probe pulse arrives before the pump, encounters the W1 waveguide in its ground state and is transmitted in the unperturbed mode present at that frequency. However, when $\tau>2 \mathrm{ps}$, the probe arrives after the pump and encounters the W1 in its excited state. There is no longer any output, as the modes have undergone a blue-shift of $0.39 \mathrm{THz}$ and the probe pulse is at frequencies which now lie in the photonic band-gap, so it is reflected.

Around $\tau=0$, the probe pulse is actually inside the waveguide when the pump arrives. At $202 \mathrm{THz}$ the probe has a transit time through the W1 waveguide of around $2.5 \mathrm{ps}$ (as derived from the measured group delay in fig. 4 (b)), and so 
the $1.3 \mathrm{ps}$ pulse is entirely contained within the waveguide when the pump arrives, and it is output at a frequency 0.3 $\mathrm{THz}$ higher than the input pulse. Figure 5 (c) shows a cut of the spectra at $\tau=-5 \mathrm{ps}$ (red dots) and $\tau=0$ (blue dots) - the arrival of the pump pulse causes the disappearance of the original input pulse and its replacement by a similar pulse blueshifted by $0.3 \mathrm{THz}$. From fig. 5 (c) it can also be seen that the efficiency of the process is high, at over $80 \%$.
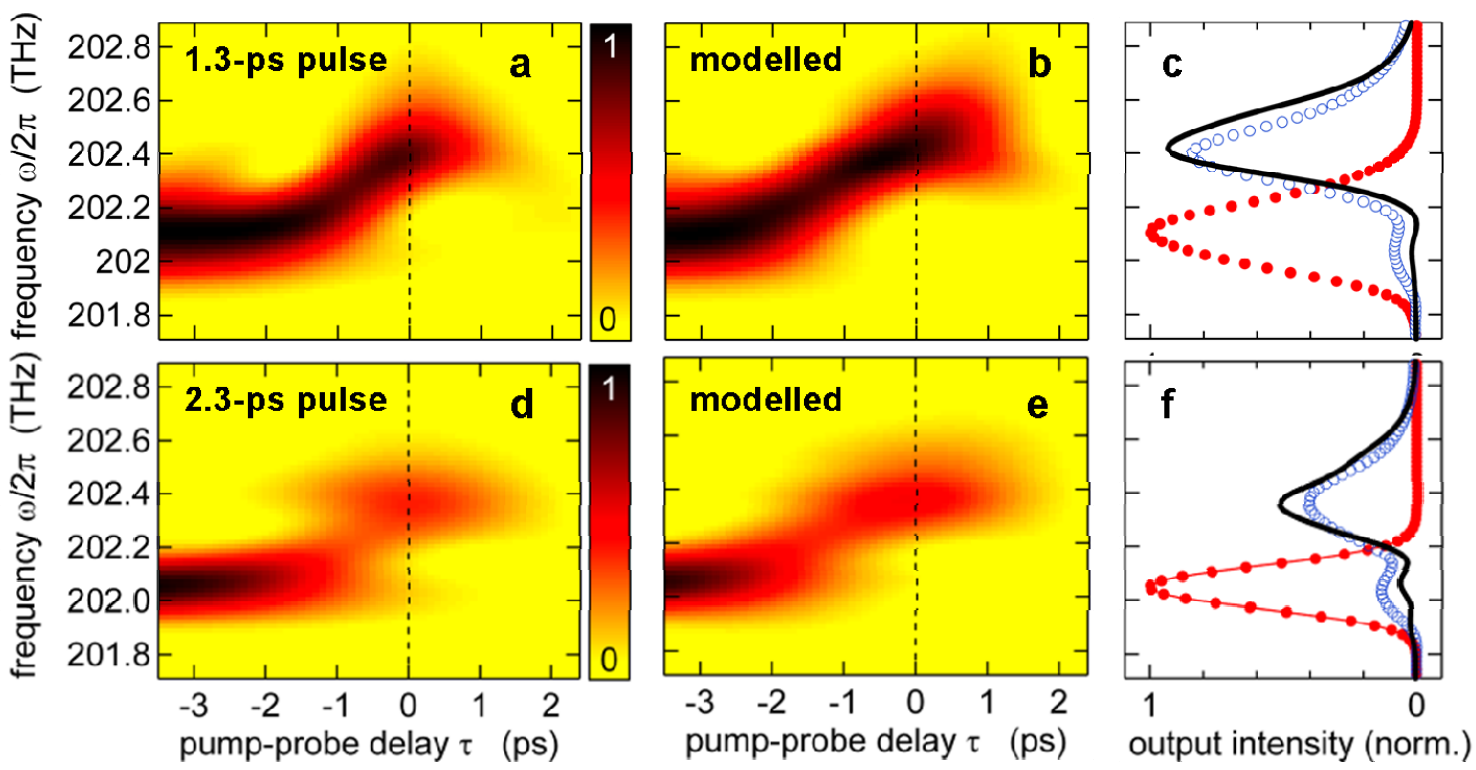

Figure 5. Measured (a) and calculated (b) output intensity spectra from the W1 waveguide as a function of pump-probe delay $t$ when a 1.3 ps long pulse centred at $202 \mathrm{THz}$ is used as input. The calculations in (b) use an analytic model with the assumption of adiabaticity, as described in ref [13]. (c) Measured (dots) output spectra at $\tau=-5 \mathrm{ps}$ (red) and $\tau=0$ (blue). The black line shows the calculated result for $\tau=0$. (d-f) Same as (a-c), but for a 2.3 ps ong input-pulse centred at $202 \mathrm{THz}$.

This high efficiency is contingent upon the maximal overlap of pump and probe pulses in the W1 waveguide. If the frequency of the probe pulse is raised, thereby decreasing its group delay [see fig. 4 (b)] and increasing its transit time, the efficiency of the process drops. A similar drop is seen if the length of the probe pulse is increased whilst the centre frequency is held constant, as demonstrated in fig. 5 (d) and (f), which shows the output if a 2.3 ps pulse is used.

To better understand the frequency conversion process, we have also analysed it using a simple - yet rigorous analytical theory. Details can be found in ref. [13], but here we note that the key assumption of the theory is that there is no coupling to any other modes - i.e. that the process is adiabatic. The results of the adiabatic theory are shown in fig. 5 (b) and (e) for the $1.3 \mathrm{ps}$ and $2.3 \mathrm{ps}$ input pulses respectively, and also by the solid lines in fig. 5 (c) and (f). The excellent agreement of this adiabatic theory with the experimental results confirms the adiabaticity of the process. The small difference in the predicted and measured efficiency of the conversion most likely arises from the loss due to absorption of the pulse by the generated free carriers - a process which is not taken into account in the analytic model.

\section{CONCLUSION}

We have performed ultrafast pump-probe experiments on a silicon photonic crystal waveguide in order to demonstrate efficient and adiabatic frequency conversion. The absorption by the silicon of a $150 \mathrm{fs}$ pump-pulse at $800 \mathrm{~nm}$ focused onto the top of a W1 waveguide generates free electron-hole pairs, which cause the abrupt decrease of the refractive index and the blue-shifting of the eigenfrequencies of the waveguiding mode. If this occurs when a telecommunications pulse is inside the waveguide, the pulse is adiabatically shifted in frequency along with the modes. In this way we have demonstrated the $0.3 \mathrm{THz}$ conversion of a 1.3 ps input-pulse with $80 \%$ efficiency in a waveguide just $19 \mu \mathrm{m}$ long. We showed that the high efficiency depends on the spatial compression of the pulse given by the slow-light modes. 


\section{ACKNOWLEDGEMENTS}

We acknowledge funding through the EU FP6-FET "SPLASH" project. This work is also part of the research program of FOM, which is financially supported by the NWO.

*daryl.beggs@st-andrews.ac.uk; phone +44 (0) $1334 \quad 46$ 7336; http://www.st-andrews.ac.uk/microphotonics/ 


\section{REFERENCES}

1. Reed, G. T., "The optical age of silicon," Nature 427, 595-596 (2004).

2. Almeida, V. R., Barrios, C. A., Panepucci, R. R. and Lipson, M. "All-optical control of light on a silicon chip," Nature 431, 1081 (2004).

3. Liu, A., Liao, L., Rubin, D., Nguyen, H., Ciftcioglu, B., Chetrit, Y., Izhaky, N. and Paniccia, M., "High-speed optical modulation based on carrier depletion in a silicon waveguide," Opt. Express 15(2), 660-668 (2007)

4. Liu, A., Jones, R., Liao, L., Samara-Rubio, D., Rubin, D., Cohen, O., Nicolaescu, R. and Paniccia, M., "A high speed silicon optical modulator based on a metal-oxide-semiconductor capacitor," Nature 427, 615-618 (2004).

5. Xu, Q., Schmidt, B., Pradhan, S. and Lipson, M., "Micrometre-scale silicon electro-optic modulator," Nature 435, 325-327 (2005).

6. Lipson, M., "Silicon photonics: the optical spice rack," Electron. Lett., 45(12), doi: 10.1049/el.2009.1232, (2009)

7. Green, W. M. J., Rooks, M. J., Sekaric, L., and Vlasov, Y. A., "Ultra-compact, low RF power, $10 \mathrm{~Gb} / \mathrm{s}$ silicon Mach-Zehnder modulator,”Opt. Express 15(25), 17106 (2007).

8. Vlasov, Y., Green, W. M. J., and Xia, F., "High-throughput silicon nanophotonic wavelength-insensitive switch for on-chip optical networks," Nature Photonics 2, 242-246 (2008)

9. Notomi, M., Shinya, A., Mitsugi, S., Kira, G., Kuramochi, E. and Tanabe, T., "Optical bistable switching action of Si high-Q photonic-crystal nanocavities,” Opt. Express 13, 2678-2687 (2005).

10. Beggs, D. M., White, T. P., O'Faolain, L. and Krauss, T. F., "Ultracompact and low power optical switch based on silicon photonic crystals," Opt. Letters 33(2), 147-149 (2008).

11. Beggs, D. M., White, T. P., Kampfrath, T., Kuipers, K., Krauss, T. F., "Slow-light photonic crystal switches and modulators" in Silicon Photonics V, edited by Joel A. Kubby, Graham T. Reed, Proceedings of SPIE Vol. 7606 (SPIE, Bellingham, WA 2010) 76060N.

12. Kampfrath, T., Beggs, D. M., White, T. P., Burresi, M., van Oosten, D., Krauss, T. F. and Kuipers, L., "Ultrafast rerouting of light via slow modes in a nano-photonic directional coupler," Appl. Phys. Lett. 94(24), 241119 (2009).

13. Kampfrath, T., Beggs D. M., White, T. P., Melloni, A., Krauss, T. F. and Kuipers, L., "Ultrafast adiabatic frequency shifting of slow light in a photonic crystal," Phys. Rev. A, in print.

14. Chutinan, A. and Noda, S., "Waveguides and waveguide bends in two-dimensional photonic crystal slabs," Phys. Rev. B 62(7), 4488-4492 (2000).

15. Notomi, M., Yamada, K., Shinya, A., Takahashi, J., Takahashi, C., Yokohama, I., "Extremely large group-velocity dispersion of line-defect waveguides in photonic crystal slabs," Phys. Rev. Lett. 87(25), 253902 (2001).

16. McNab, S. J., Moll, N., Vlasov, Y. A., "Ultra-low loss photonic integrated circuit with membrane-type photonic crystal waveguides," Optics Express 11(22), 2927-2939 (2003).

17. Beggs, D. M., O'Faolain, L., and Krauss, T. F., "Accurate determination of the functional hole size in photonic crystal slabs using optical methods," Photonics and Nanostructures - Fundamentals and Applications 6, 213-218 (2008).

18. Notomi, M. and Mitsugi, S., "Wavelength conversion via dynamic refractive index tuning of a cavity," Phys. Rev. A 73, 051803 (2006).

19. Preble, S. F., Qianfan, X. and Lipson, M., "Changing the colour of light in a silicon resonator," Nature Photonics 1, 293-296 (2007).

20. O'Faolain, L., Yuan, X., McIntyre, D., Thoms, S., Chong, H., De la Rue, R. M. and Krauss, T. F., "Low-loss propagation in photonic crystal waveguides," Electron. Lett. 42(25), 1454-1455 (2006).

21. White, T. P., O'Faolain, L., Li, J., Andreani, L. C. and Krauss, T. F., "Silica-embedded silicon photonic crystal waveguides," Opt. Express 16(21), 17076-17081 (2008).

22. Beggs, D. M., White, T. P., Cairns, L., O'Faolain, L. and Krauss, T. F., "Ultrashort photonic crystal optical switch actuated by a microheater," Photonics Technol. Lett. 21(1), 24-26 (2009).

23. Kampfrath, T., Beggs, D. M., Krauss, T. F. and Kuipers, L., "Complete response characterization of ultrafast linear photonic devices," Optics Letters 34(21), 3418-3420 (2009). 